\title{
Transformaciones y prospectiva del paisaje en el páramo de Paja Blanca, Nariño, Colombia
}

\author{
TRANSFORMATION AND PROSPECTIVE IN THE LANDSCAPE OF \\ PARAMO PAJA BLANCA, NARIÑO, COLOMBIA \\ TRANSFORMAÇÕES E PREVISÃO DA PAISAGEM NO PÁRAMO DE PAJA \\ BLANCA, NARIÑO, COLÔMBIA
}

Para citar este artículo: Muñoz-Guerrero, D. A. (2017). Transformaciones y prospectiva del paisaje en el páramo de Paja Blanca, Nariño, Colombia. Perspectiva Geográfica, 22(2),47-66. doi: 10.19053/01233769.7598
DIEGO ANDRÉS Muñoz-Guerrero ${ }^{1}$

Recepción:

24 de enero de 2017

Evaluación:

7 de julio de 2017

Aprobación:

24 de julio de 2017

\section{Resumen}

La presente investigación evalúa las trasformaciones en el paisaje ocurridas en el páramo de Paja Blanca, Nariño, Colombia, en el periodo 1984-2013; así mismo, a través de un análisis descriptivo y regresiones logísticas, se establecen los principales factores socioeconómicos y biofísicos que han sido determinantes en las transformaciones o cambios en el periodo mencionado. Mediante el enfoque de Redes Neuronales Artificiales (RNA) se hicieron simulaciones del paisaje para los próximos 30 años. Los resultados indican que las transformaciones más importantes están determinadas por el cambio de bosque natural fragmentado a mosaico de pastos y cultivos, con influencia de las variables distancia de vías, distancia a los ríos y también por el crecimiento poblacional; lográndose determinar una tasa de deforestación anual de 16 has/año. Las simulaciones futuras predicen un comportamiento similar, con pérdidas importantes de bosque natural fragmentado y también una reconversión de áreas de cultivos a bosques naturales.

1 Ingeniero agroforestal de la Universidad de Nariño, magíster en Agroforestería tropical del Centro Agronómico Tropical de Investigación y Enseñanza. Doctor en Geografía del Convenio UPTC-IGAC. Profesor Universidad de Nariño. damg30@hotmail.com 
Palabras clave: análisis espacial, análisis multitemporal, cambios de uso del suelo, redes neuronales artificiales.

\section{Abstract}

The present investigation evaluates the transformations in the landscape occurred in the paramo of Paja Blanca, Nariño, Colombia, in the period 1984-2013; Likewise, through a descriptive analysis and logistic regressions, we established the main socioeconomic and biophysical factors that have been determinant in the transformations or changes in the mentioned period. Through the Artificial Neural Networks (RNAs) approach, landscape simulations were also made for the next 30 years. The results indicate that the most important transformations are determined by the change from fragmented natural forest to mosaic of pastures and crops, influenced by the variables: distance of roads, distance to rivers and also by population growth; Achieving an annual deforestation rate of 16 ha/year. Future simulations predict similar behavior, with significant losses of fragmented natural forest, and also a reconversion of crop areas to natural forests.

Keywords: spatial analysis, multi-temporal analysis, changes in land use, artificial neural networks.

\section{Resumo}

A presente pesquisa avalia as transformações na paisagem acontecidas no Páramo de Paja Blanca, Nariño, Colômbia, no período 1984-2013; do mesmo modo, através de uma análise descritiva e regressões logísticas, os principais fatores socioeconômicos e biofísicos que foram decisivos nas transformações ou mudanças no período mencionado são estabelecidos. Através da abordagem das Redes Neurais Artificiais (RNAs), simulações de paisagem foram realizadas para os próximos 30 anos. Os resultados amostram que as transformações mais importantes são determinadas pela mudança de bosque natural fragmentado ao mosaico de pastagens e lavouras, com influência das variáveis distância de vias, distancia aos rios e também pelo crescimento populacional; podendo determinar uma taxa de desmatamento anual de 16 ha/ano. As simulações futuras predizem um comportamento similar, com perdidas importantes de bosque natural fragmentado e também uma reconversão de áreas de lavouras para florestas naturais.

Palavras chave: análise espacial, análise multitemporal, câmbios de uso do solo, redes neuronais artificiais. 


\section{Introducción}

El paisaje, según Bertrand (1968), es una porción del espacio caracterizado por un tipo de combinación dinámica de elementos geográficos diferenciados, abióticos, bióticos y antrópicos; es también altamente dinámico y mayormente cambiante a medida que se aleja de un punto de equilibrio en términos económicos, productivos o ambientales. Frente a lo anterior, resulta claro que, para el entendimiento de la dinámica del paisaje, los estudios deben enmarcarse dentro de un contexto temporal, que permita evaluar y entender sus niveles de transformación. Las transformaciones del paisaje, según Aldana (2011) se refieren a la variación que el paisaje puede sufrir en su estructura, composición y funcionamiento durante un periodo de tiempo determinado, el cual está dado por el cambio de estado de una o más variables; estos cambios pueden darse por múltiples factores. Al respecto, Gómez-Zotano y Riesco Chueca (2010), manifiestan que dichos cambios se agudizan durante un número reducido de periodos cortos, separados por largos periodos de estabilización.

Uno de los paisajes que a nivel mundial resulta importante explorar y analizar por sus funciones ecológicas y por su importancia social son los páramos, los cuales a pesar de estar inmersos en modelos de protección como son los parques nacionales, las gestiones políticas y administrativas quizá no han sido lo suficientemente eficaces para detener la intervención antrópica; lo cual implicado unas transformaciones constantes, y con ello repercusiones negativas ambientales y sociales. Un ejemplo de estos páramos es el de Paja Blanca, ubicado en el departamento de Nariño, Colombia, decretado como área protegida desde el año 1989 y como Parque Natural Regional en el año 2015.
Corponariño (2010) afirma que en el páramo de Paja Blanca son 113 hectáreas que se deforestan anualmente y que a pesar de que esta entidad ha realizado tres planes de manejo diferentes, y ha venido implementando desde 1997 diversos mecanismos encaminados a detener el avance del sector agropecuario, como son proyectos productivos pecuarios, reforestaciones con especies nativas, fomento de estufas a gas, letrinización, entre otros, los resultados no han sido los mejores ni contundentes; los procesos de transformación del paisaje, reflejados en los cambios de coberturas, aún continúan y con ello las alteraciones ambientales, como es la reducción de los caudales que alimentan los 45 acueductos y los cinco distritos de riego de los siete municipios que lo rodean.

En la actualidad, se han desarrollado varios métodos de análisis y modelamiento espacial para explicar y predecir los cambios que se producen o se producirán en un determinado paisaje dependiendo del comportamiento de las coberturas en el tiempo y espacio. Al respecto, Sandoval y Oyarzun (2004) manifiestan que la modelación espacial del cambio en el uso del suelo se deriva de la combinación y uso de técnicas cartográficas, Sistemas de Información Geográfica y modelos estadísticos multivariantes. En este sentido, en el presente estudio se usaron metodologías de percepción remota y modelamiento espacial para explicar las transformaciones del paisaje del páramo de Paja Blanca en los últimos 30 años, así como su prospectiva para los 30 próximos, de continuar con las tendencias actuales; información que será muy útil para los procesos de toma de decisiones de las entidades encargadas de su administración y manejo.

\section{2. Área de estudio}

La zona de estudio corresponde al páramo de Paja Blanca, que se ubica en el departamento de Nariño, 
en la parte central del nudo de los Pastos entre las coordenadas $0^{\circ} 50^{\prime}-1^{\circ} 06^{\prime}$ de latitud norte y $77^{\circ}$ $45^{\prime}-77^{\circ} 25^{\prime}$ de longitud oeste (Figura 1). Según el documento elaborado por Corponariño en el año 2010, denominado "Declaratoria del Parque Natural Regional Páramo de Paja Blanca, Territorio Sagrado del pueblo de los Pastos", tiene un área de 4640 hectáreas, superficie tomada en cuenta para este estudio. Presenta una distribución altitudinal entre los 3000 y 3600 m s.n.m. La cumbre del páramo de Paja Blanca corresponde a un cráter antiguo con productos volcánicos extensos. Según Corponariño (2010), corresponde a un sistema aislado del complejo de páramos suroccidentales del departamento de Nariño; tal situación y la altura de los páramos circunvecinos (Chiles, Cumbal y Azufral) han condicionado una estructura ecosistémica de características atípicamente más xéricas, con menos influencia húmeda o perhúmeda de las corrientes pacíficas y amazónicas respecto a los otros páramos suroccidentales. Las temperaturas medias anuales oscilan entre $\operatorname{los} 10,2^{\circ} \mathrm{C}$ y $11,6^{\circ} \mathrm{C}$, y las precipitaciones entre 1094 y 1242 mm. (Corponariño, 2007a). Este páramo cubre los sectores altos de los municipios de Pupiales, Sapuyes, Ospina Iles, Guachucal, Contadero y Gualmatán. En relación con los eventos volcánicos, ha recibido, por su relativa cercanía con el sistema del volcán Azufral y en diferentes períodos geológicos, grandes cantidades de material de tipo volcánico, que en la actualidad se puede hacer evidente en los cortes y capas deposicionales presentes en la geología del páramo Paja Blanca, extendiéndose sobre todo hacia el sector nororiente, en Sapuyes (Corponariño, 2007b).

\section{Metodología}

\subsection{Procesamiento digital de imágenes}

Para establecer las coberturas del año 1984, se utilizaron imágenes satelitales Landsat 7 TM (Path 010, Row 059). Para el año 2013, se adquirieron imágenes del sensor Aster mediante el aplicativo de la NASA disponible en internet (https://wist.echo.

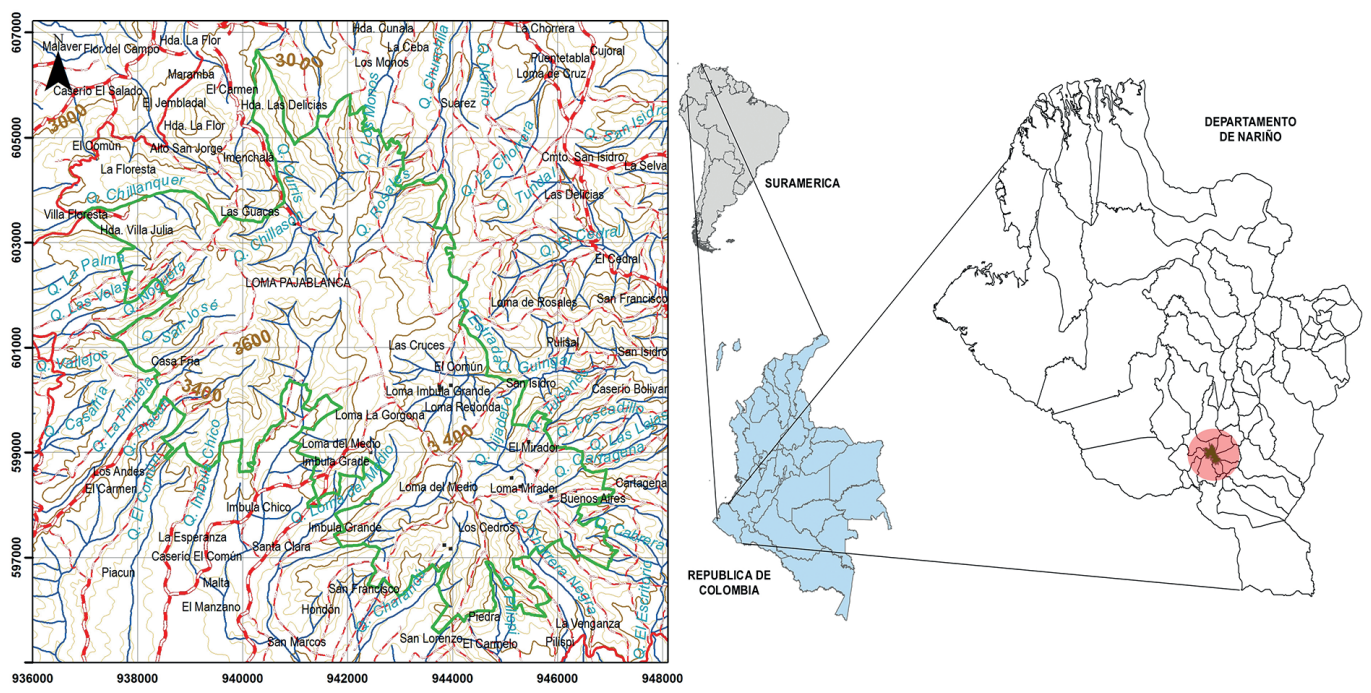

FIGURA 1.

Localización área de estudio

Fuente: Elaboración propia. 
nasa.gov/). Para la calibración geométrica de las imágenes, se utilizó el programa Erdas 2011, en el cual mediante la recolección de puntos GPS en campo con el actual Datum de la cartografía colombiana (MAGNA-Sirgas), se realizó la georreferenciación $($ RMS $<1)$. La calibración radiométrica y atmosférica, proceso que tiene como objetivo corregir problemas mecánicos en el sensor que generan valores erróneos en píxeles concretos y eliminar las distorsiones que la atmósfera introduce en los valores de radiancias que llegan al sensor desde la superficie terrestre, implicó la conversión de niveles digitales (ND) a radiancias (Chuvieco, 1996); conversión de niveles de radiancia a reflectividad aparente o reflectancia (Wooster, 1996; Thome, Palluconi, Takashima y Masuda, 1998; Chander y Markham, 2003). La calibración atmosférica se hizo a través del modelo propuesto por Chávez (1996), el cual consiste en detectar en una banda el valor mínimo (m), con el fin de sustraer a cada ND de la imagen dicho valor.

\subsection{Clasificación de las coberturas}

Después de los anteriores procedimientos, en el programa Erdas 2011 se procedió a realizar una clasificación de las coberturas presentes en cada año; para ello se aplicó la metodología de clasificación supervisada, la cual exige una misma leyenda para las dos fechas, con el propósito de que sean realmente comparables. La clasificación de las categorías de uso se efectuó teniendo en cuenta la metodología Corine Land Cover; y todo el proceso de vectorización así como la producción final de los mapas de coberturas se realizaron en el programa ArcGIS 10. Las categorías utilizadas fueron: bosque natural denso (BND), bosque natural fragmentado (BNF), mosaico de pastos y cultivos (MPC) y coberturas de páramo $(\mathrm{PA})$.

\subsection{Dinámica de los cambios de coberturas del suelo ocurridos entre 1984 y 2013}

Una vez obtenidos los mapas de usos y coberturas, se realizó un análisis estadístico descriptivo espacio-temporal de los cambios ocurridos entre 1984 y 2013, utilizando para ello el módulo Land Change Modeler (LCM) del programa IDRISI ANDES; este módulo permite realizar de manera rápida un análisis de las ganancias y pérdidas de superficies experimentadas por los distintos usos y coberturas de suelo, sus cifras netas y transiciones; datos que se usaron para construir una matriz de tabulación cruzada, que se describe como una tabla con arreglos simétricos que contiene en uno de sus ejes las categorías de uso de suelo y vegetación del año base y en el otro eje las mismas categorías pero del segundo tiempo.

\subsection{Identificación y análisis de los principales factores que han incidido en los procesos de transformación del paisaje}

En el presente estudio se optó por tomar las causas más relevantes que propone la literatura basadas en la caracterización de los diferentes flujos de cambio en la cobertura y el uso del suelo. De esta manera, se analizaron los factores demográficos como principales causantes de las transformaciones paisajísticas (Henríquez-Dole, 2012; Bonilla, 1985; Harthshorn, 1983; Pérez y Protti, 1978). Se tomaron datos estadísticos del Departamento Administrativo Nacional de Estadística (DANE), para con ello analizar las tendencias de crecimiento en los siete municipios que conforman el ámbito de estudio; de igual forma, los datos de crecimiento poblacional se utilizaron para generar mapas de densidad poblacional. Con el 
objetivo de averiguar si dichos valores de densidad poblacional predisponen una mayor ocurrencia de unas categorías temáticas de ocupación del suelo, antes que otras, se utilizó el estadístico Cramer's $\mathrm{V}$., cuyos valores varían entre 0 y 1 ; un valor de 0 implica independencia de los atributos y un valor de 1 denota asociación perfecta.

Considerando las recomendaciones de los anteriores autores, se realizaron también distancias euclidianas y regresiones logísticas con el fin de descubrir la relación entre la estructura vial y las viviendas, con las áreas que se transformaron durante 1984 y 2013. Los mapas de distancias euclidianas, que corresponde a la distancia desde las vías y viviendas a cualquier punto de las zonas de estudio, se construyeron con el comando "Distance" del programa Idrisis Selva. La regresión logística es un método similar a la regresión lineal múltiple, pero adaptado a modelos en donde es condición necesaria que la variable a explicar sea binaria, pudiendo tomar únicamente valores de 0 y 1 (Pineda-Jaimes, Bosque-Sendra, Gómez-Delgado y Plata-Rocha, 2009). El estadístico utilizado para evaluar el modelo de regresión fue el ROC (Relative Operating Charasteric), sus valores oscilan entre 0 y 1 , de modo que valores de 0,5 o menores no indican ningún grado de correlación y valores cercanos a 1 indican un buen ajuste entre variables; todo el proceso se realizó con el módulo Logisticreg del programa Idrisi Selva.

Al igual que las variables socioeconómicas, también se hicieron regresiones logísticas para encontrar relaciones entre algunas variables físico-bióticas y las transformaciones presentadas en los últimos 30 años. Las variables que se escogieron fueron: el relieve, las pendientes y la distancia a los ríos. Para el relieve, se utilizó un modelo digital de terreno de 12,5 metros de resolución, proveniente del sensor
ALOS PALSAR, las pendientes se obtuvieron del modelo digital de terreno calculadas en porcentaje con el comando "SLOPE” del programa Idrisi Selva, y para la distancia a los ríos se usaron distancias euclidianas.

\subsection{Simulación}

La simulación de los paisajes del páramo de Paja Blanca para los próximos 30 años se realizó con base en el enfoque de Redes Neuronales Artificiales (RNA) (Isasi y Galván, 2004), las cuales son estructuras de mapeo no lineal basadas en el funcionamiento del cerebro humano. Se utilizó para ello el módulo Land Change Modeler (LCM) que viene incorporado en el programa Idrisi Selva. Dicho enfoque requirió efectuar los siguientes pasos:

- Selección y análisis de variables de transición: con base en los resultados del acápite anterior, se seleccionaron las variables cuyo patrón espacial manifieste algún tipo de comportamiento que se crea que puede tener relación con el patrón de localización de cada uno de los cambios de coberturas que se identificaron. Las variables seleccionadas fueron sometidas a la prueba Cramer's V para calcular la fuerza de la relación entre estas y la distribución de las categorías de usos del suelo de la fecha más reciente (2013). Si bien un alto índice Cramer's V no garantiza una buena modelización, un bajo índice sí es un indicativo de la conveniencia de no incluirlas en la modelación (Clarks Labs, 2006). LCM permite realizar de manera muy fácil la prueba de Cramer's V, para tomar la decisión de incluir o no una variable en el modelo.

- Identificación de las transiciones potenciales: en este paso, se definieron en primera instancia cuáles son las transiciones o cambios más impor- 
tantes, para lo cual se escogieron aquellas que afectarían a más de dos hectáreas de superficie. Con las transiciones definidas, en el módulo LCM de Idrisi Selva fueron agrupadas en un submodelo, al cual se agregaron las variables de transición, para posteriormente generar mapas potenciales de transición, que son la plasmación espacio-temporal del potencial de cambio que oscilan de 0 a 1 , indicando el potencial que cada píxel presenta para cambiar de una categoría de cobertura a otra. Este proceso se basa en el entrenamiento de las redes neuronales a través de la minimización del error cuadrático medio, variando la tasa de aprendizaje, el número de nodos en la capa intermedia, el número total de iteraciones y la revisión gráfica de la evolución del error cuadrático medio.

- Estimación de la cantidad de cambio y localización de los cambios: después de obtener las matrices de transición de cada categoría de cobertura, el módulo LCM de Idrisi predice los cambios para los próximos 30 años (2042). Este modelo, en primer lugar, genera una matriz de probabilidad de cambio para el periodo asignado, luego ofrece dos clases de predicción: una imagen con la predicción de un escenario específico y una imagen con índices de la vulnerabilidad al cambio. La primera se presenta con la clasificación final basado en un modelo de localización competitiva del suelo, y la segunda representa las áreas que presentan las mejores condiciones para cambiar en una escala de 0 a 1 (Henríquez-Dole, 2012).

\subsection{Validación}

Para validar el modelo obtenido, se hizo una simulación para el año 2016, obteniéndose un mapa raster de coberturas para dicho año; posteriormente se realizó en campo un muestreo aleatorio simple, en el que se seleccionaron al azar 50 puntos donde se registró su posición GPS y el tipo de cobertura presente. Estos puntos fueron ingresados al programa ArcGIS 10 con sus respectivas coordenadas geográficas y sus atributos de coberturas observadas; posteriormente se ingresó el mapa raster de las coberturas simuladas para el año 2016, y se usó la herramienta "Extract Values to Points" de ArcGIS para capturar las coberturas simuladas. Como resultado se obtiene una tabla donde cada punto contiene el tipo de cobertura observada y simulada. Con estos puntos se construyó una matriz de confusión, que, según Congalton, Lunetta, Fenstermaker, Jensen, McWire y Tinney (1991), consiste en la colocación de filas y columnas relacionadas con las categorías de uso o cobertura relacionada con el mapa. En las columnas se ubican las categorías deducidas por la simulación, mientras que en las filas se colocan las clases de referencia (verdad del terreno). El error asociado a cada columna se denomina "error de inclusión o comisión" y representa aquellas áreas que fueron asignadas a dicha categorías sin pertenecer a ella. En las filas se presentan las categorías verdaderas. El error asociado a cada fila se denomina "error de exclusión u omisión" y representa aquellas áreas que no fueron asignadas a dicha categoría aun cuando pertenecían a ella. También se evaluó la clasificación con el índice de Kappa, que es otra forma altamente difundida de medir la exactitud de un mapa. Conocida también como " $k$ ", puede medir la exactitud de manera más precisa que la matriz de confusión, porque incluye dentro del cálculo todos los valores de la matriz y no solamente sus extremos; su fórmula según Cohen (1960) es la siguiente:

$$
\hat{K}=\frac{n \sum a_{i i}-\sum^{l}\left(a_{i .} a_{. i}\right)}{n^{2}-\sum_{i=1}^{l}\left(a_{i .} a_{i .}\right)}
$$


Donde:

$\mathrm{i}$ = dimensión de la matriz (número de clases);

aii = número de observaciones en la línea i, columna i; ai. y a. $i=$ total marginal de línea $i$ y de columna $i$, $\mathrm{n}=$ número total de observaciones

\section{Resultados y discusión}

\subsection{Dinámica de los cambios de coberturas del suelo ocurridos entre 1984 y 2013}

La Figura 2 muestra las coberturas del suelo que se obtuvieron mediante el procesamiento de las imágenes Landsat y Aster para los años 1984 y 2013.
La Tabla 1 muestra la cantidad de hectáreas que se ganaron y se perdieron en cada categoría de cobertura del suelo durante el periodo 1984-2013.

Los resultados muestran que en el periodo 19842013 fueron 768 ha que se transformaron entre las diferentes categorías. Las transformaciones más críticas corresponden al bosque natural fragmentado (BNF), puesto que en dicho periodo se perdieron 394 hectáreas que pasaron a ser dedicadas en su mayoría (348 has) a mosaico de pastos y cultivos (MPC). Cabrera y Ramírez (2014) sostienen al respecto que el incremento de las actividades antrópicas en los páramos ha sido reportado desde mucho tiempo atrás, y que en la actualidad los MPC es el tipo de cobertura de uso antrópico que más predominan en los páramos colombianos; esto se

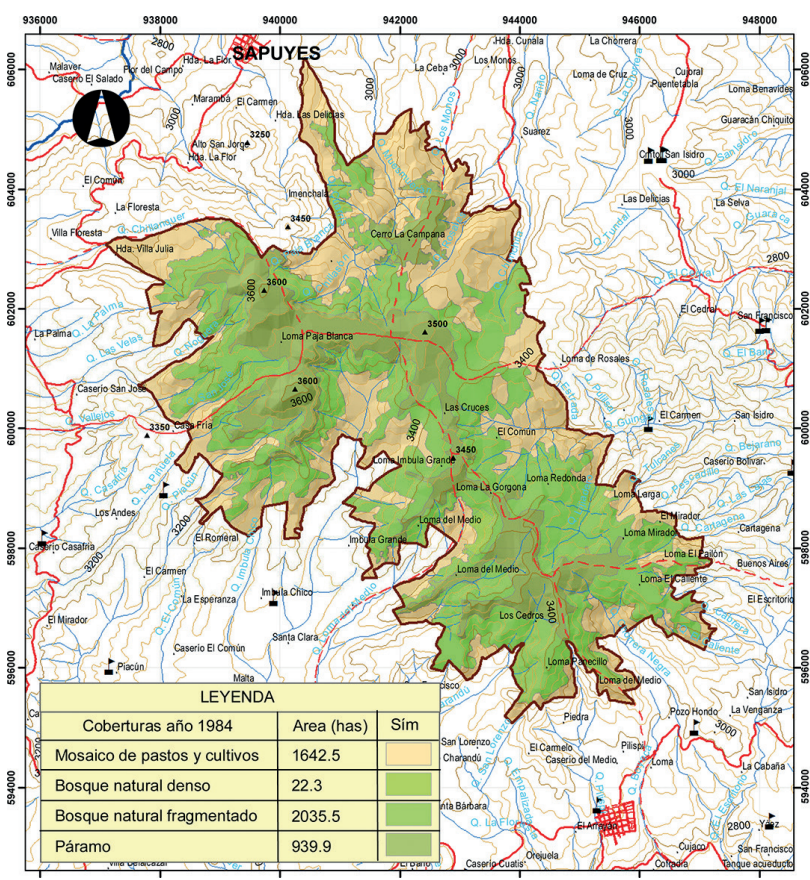

a) Coberturas año 1984

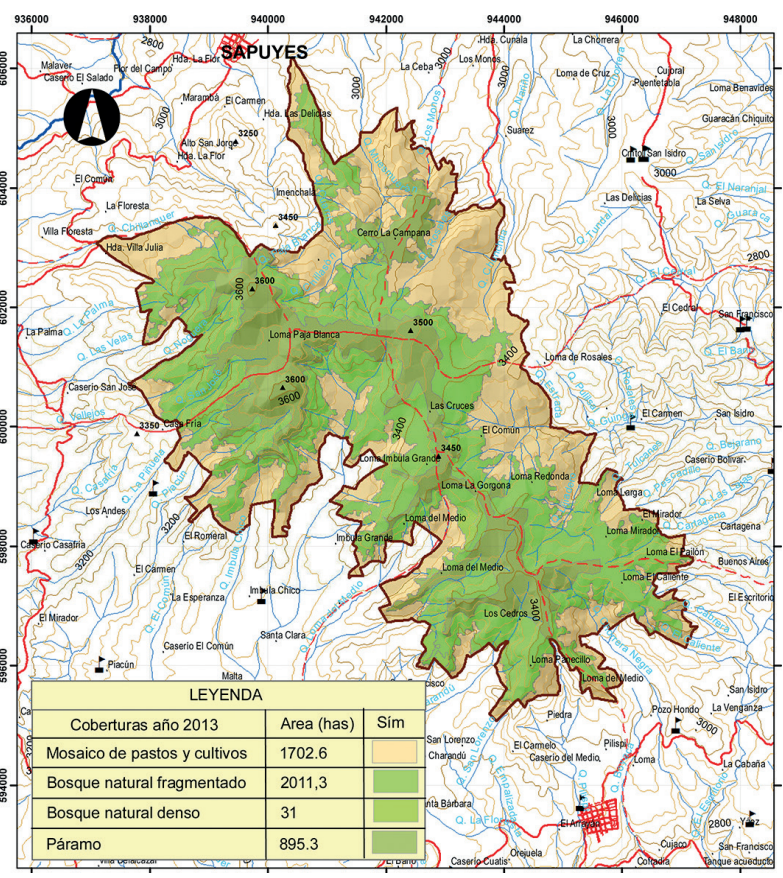

b) Coberturas año 2013

FIGURA 2.

Coberturas del suelo en el páramo de Paja Blanca: años 1984 y 2013

Fuente: Elaboración propia. 
TABLA 1. TABULACIÓN CRUZADA CON GANANCIA, PÉRDIDAS Y PERSISTENCIA DE COBERTURAS DEL SUELO EN EL PÁRAMO PAJA BLANCA PARA EL PERIODO 1984-2013

\begin{tabular}{lcccccc}
\multicolumn{1}{c}{$\mathbf{1 9 8 4 / 2 0 1 3}$} & BND & BNF & MPC & PA & Total & Pérdidas \\
\hline Bosque natural denso (BND) & 18,5 & 0,0 & 3,8 & 0 & 22,3 & 3,8 \\
Bosque natural fragmentado (BNF) & 12,3 & 1641,2 & 348,8 & 33,2 & 2035,5 & 394,3 \\
Mosaicos de pastos y cultivos (MPC) & 0,2 & 292,3 & 1350,0 & 0 & 1642,5 & 292,5 \\
Páramo (PA) & 0 & 77,8 & 0 & 862,1 & 939,9 & 77,8 \\
Total & 31,0 & 2011,3 & 1702,6 & 895,3 & 4640,2 & \\
Ganancias & 12,5 & 370,1 & 352,6 & 33,2 & & \\
\hline
\end{tabular}

Fuente: Elaboración propia.

debe, según Morales-Betancourt y Estévez (2006), a que, a diferencia de las selvas húmedas, los páramos pueden ser adaptados fácilmente para el cultivo y la ganadería con solo la quema de predios en los que el fuego se esparce fácilmente por acción de los fuertes vientos.

Hubo también 292 hectáreas de MPC que mediante procesos de protección y regeneración natural pasaron a ser en el año 2013 bosque natural fragmentado (BNF).

En bosque natural denso (BND), se presentaron más ganancias que pérdidas, que desde el punto de vista ambiental es bastante positivo, reflejando quizá que las gestiones de manejo del páramo por parte de Corponariño han logrado que las comunidades preserven los bosques fragmentados hasta el punto de que lleguen a un estado de BND.

Las coberturas de páramo (PA), ya sean herbáceas o arbustivas, sufrieron pocas transformaciones, presentándose casi 78 hectáreas de páramo que pasaron a ser BNF.

Cabrera y Ramírez (2014), como producto del resultado de la actualización del Atlas de páramos de Colombia, establecen unas categorías según el grado de intervención antrópica que han sufrido; de acuerdo con esto, Paja Blanca presenta un grado de transformación baja, ya que las coberturas transformadas antrópicamente representan el 7,5\% del total del páramo; siendo similar a otros páramos colombianos clasificados dentro de esta categoría y que presentan porcentajes casi similares como son: Guanacas-Puracé-Coconucos (7,34\%), Chilí-Barragán (6,89\%) y Chingaza (6,72\%). Según estos autores, en estos páramos los esfuerzos de restauración deberían enfocarse a la contención y reconversión de la transformación del paisaje que contribuya a disminuir los procesos de fragmentación.

\subsection{Principales factores que han incidido en los procesos de transformación del paisaje en el páramo de Paja Blanca}

En el páramo de Paja Blanca, las comunidades interactúan directamente con los recursos naturales que ofrece este ecosistema, para solventar necesidades alimenticias y económicas; por ello, Corponariño (2007a) afirma que, en la zona de estudio, el crecimiento poblacional ha sido un factor determinante en las transformaciones del paisaje. La Figura 4 indica cómo ha sido la tendencia del crecimiento poblacional de los siete municipios que conforman el páramo de Paja Blanca desde el año 1985. 
FIGURA 4.

Crecimiento poblacional de los siete municipios que integran el páramo de Paja Blanca 1985-2013

Fuente: DANE (2013).

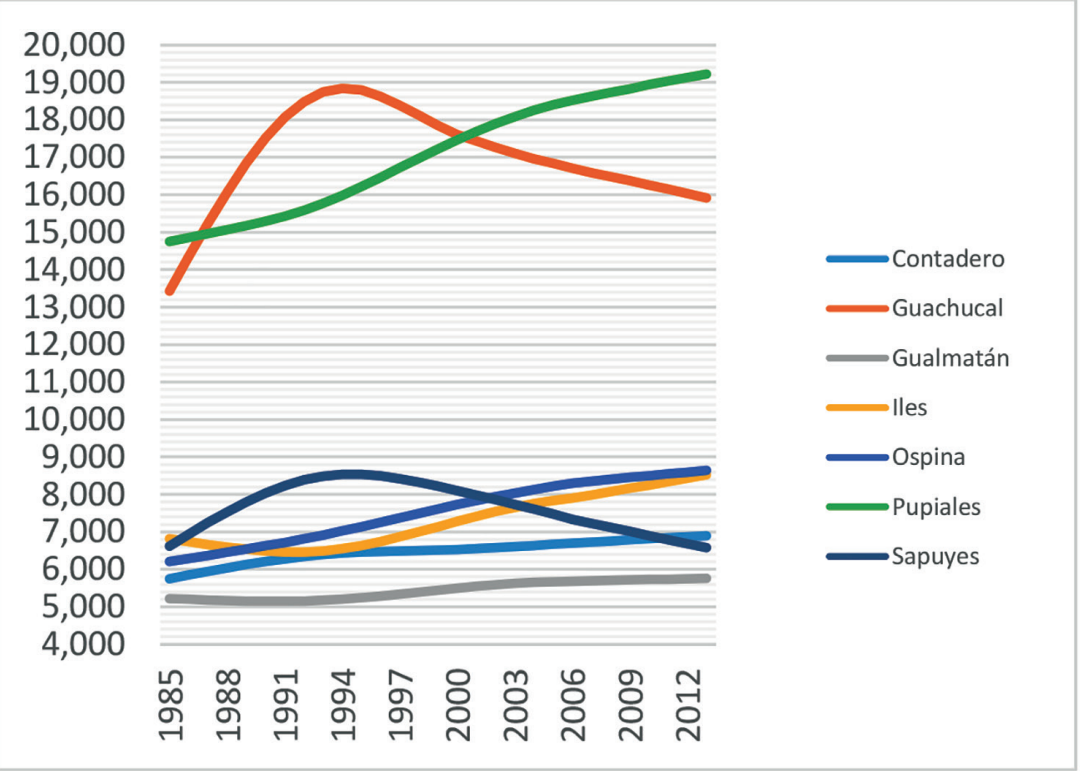

Según el censo poblacional de 1985, el total de población en los municipios del páramo de Paja Blanca fue de 58788 habitantes, representando una densidad poblacional promedio de 127 habitantes/ $\mathrm{km}^{2}$, que es bastante superior a la densidad media del departamento de Nariño (45 hab $\left./ \mathrm{km}^{2}\right)$, y mucho mayor a la media nacional $\left(36.3 \mathrm{hab} / \mathrm{km}^{2}\right)$. Los municipios de Guachucal y Pupiales presentan la mayor población, doblando su cantidad al resto de municipios, sin embargo, la mayor densidad poblacional se presenta en los municipios de Iles y Sapuyes, con 155 y $190 \mathrm{hab} / \mathrm{km}^{2}$, respectivamente. Para el posterior censo, en el año 2005, el total de población pasó a 71111 personas, es decir, hubo un aumento del 20\% (12323 habitantes), y para el 2013, el DANE proyecta una población total de 71519 habitantes.

El área de influencia directa del páramo Paja Blanca se compone de 27 veredas, con una población total de 6950 personas, que corresponde al 13,08\% del total de la población rural. Según Corponariño (2007a), sobre los 3200 m.s.n.m. habitan 305 familias, con un total de 1400 personas aproximadamente, que representa el 2,4\% del total de la población rural. De acuerdo con Corponariño (2010), el 56\% de la población que habita en el interior del área del páramo tiene 30 años o menos, y su distribución por género es de 51,06\% hombres y 48,94\% mujeres.

Posiblemente la presencia de gente en las partes altas del páramo obedezca a una situación socioeconómica relacionada con la tenencia de la tierra; las partes bajas ya han sido lo suficientemente explotadas, lo que ha obligado a algunos campesinos a buscar tierras más productivas hacia zonas de más altura, donde las condiciones edafológicas son más favorables. Al respecto, Corponariño (2007a) concluye que "la totalidad del páramo de Paja Blanca está ocupado, no existen baldíos, sus propietarios en más del 70\% habitan en el páramo y por ende de acuerdo a las condiciones económicas, sociales y culturales deben su sustento de lo que producen en el mismo" (p. 154).

Los resultados con respecto a si los valores de densidad poblacional de cada uno de los siete municipios que hacen parte de Paja Blanca predisponen una 
mayor ocurrencia de unas categorías temáticas de ocupación del suelo, antes que otras, no mostraron relación alguna; se presentaron coeficientes de Cramer's V bastante bajos, lo que indica independencia

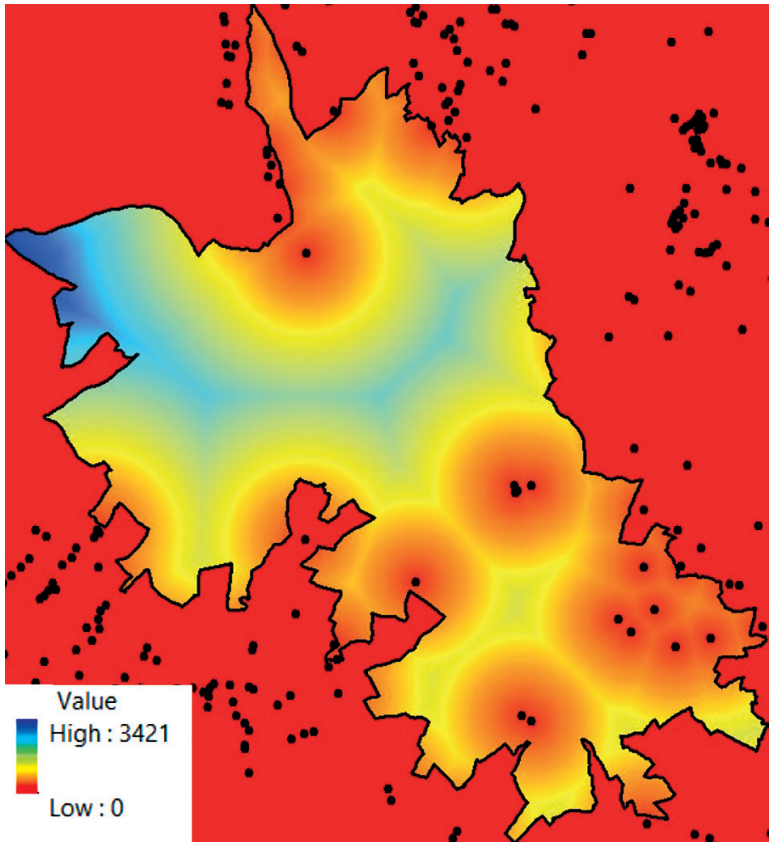

a)

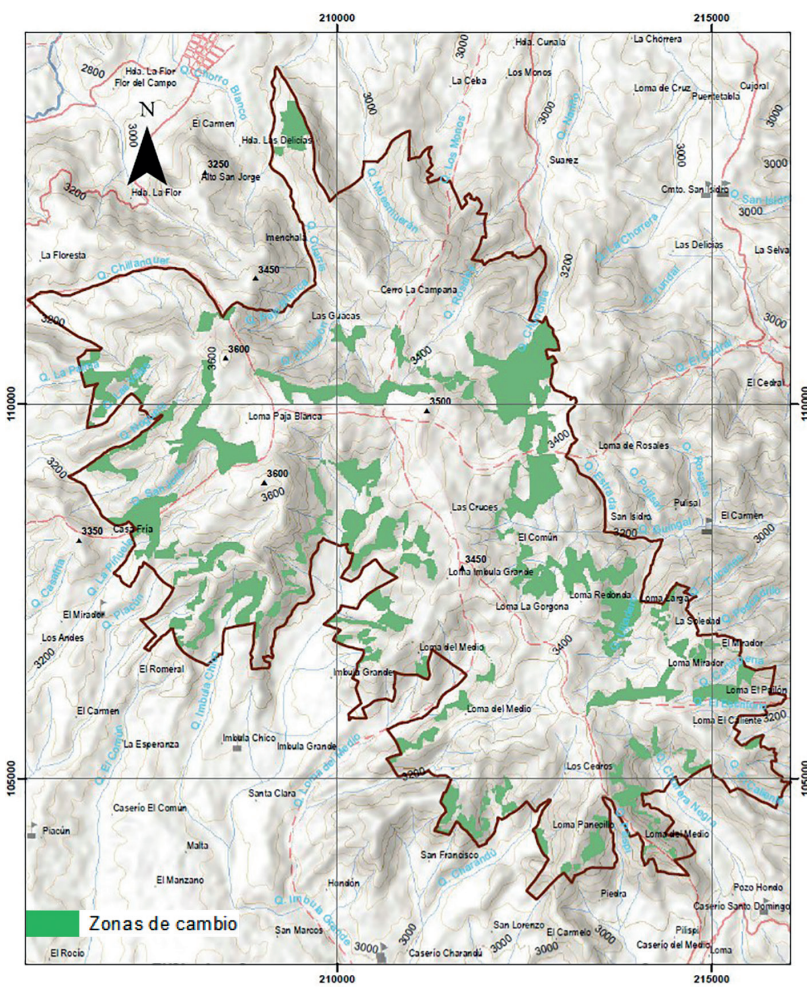

entre estas dos variables. Las regresiones logísticas entre las vías y viviendas (Figura 5) y su relación con las áreas transformadas (Figura 6) mostraron un ROC bastante alto (Tabla 2).

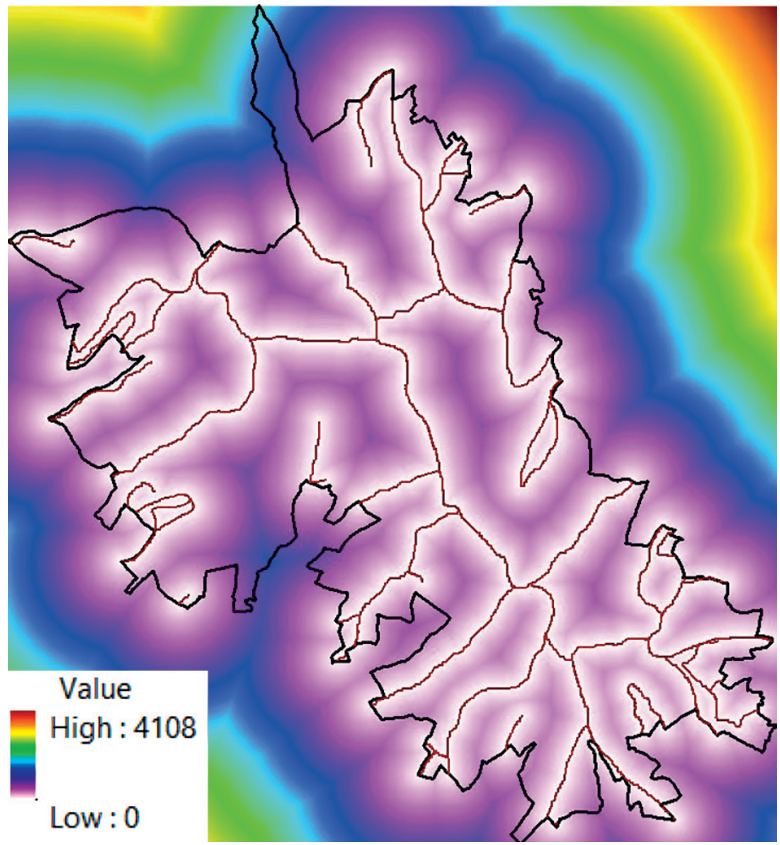

b)

FIGURA 5

Distancias euclidianas de viviendas (a) y vías (b) en el páramo de Paja Blanca

Fuente: Elaboración propia.

\section{FIGURA 6.}

Áreas transformadas o de cambio entre 1984 y 2013 en el páramo de Paja Blanca Fuente: Elaboración propia. 
TABLA 2. VALORES DE REGRESIÓN LOGÍSTICA ENTRE ÁREAS TRANSFORMADAS Y DISTANCIA A VÍAS Y VIVIENDAS EN EL PÁRAMO DE PAJA BLANCA.

\begin{tabular}{cccc} 
VARIABLES & COEFICIENTE & INTERCEPT & ROC \\
\hline Distancia a vías & $-0,00252141$ & $-1,49074172$ & 0,76 \\
Distancia a viviendas & 0,00117711 & $-3,43462289$ & 0,81
\end{tabular}

Fuente: Elaboración propia.

Los resultados indican que a pesar de mostrar un ROC alto, el comportamiento es diferente en estas variables; así, la distancia a las viviendas presenta un coeficiente positivo, es decir, un aumento en una unidad de estas distancias aumenta las áreas transformadas, lo que revela que los cambios en el paisaje no se dan necesariamente en las cercanías a las viviendas. Por el contrario, en la distancia a las vías, el coeficiente es negativo, indicando que hay más transformaciones en áreas cercanas a estas. Este comportamiento es de esperarse, pues tal como se observa en la Figura 5(b), la distribución de vías, como son caminos y carreteras, atraviesan casi toda el área del páramo, facilitando de esta manera la accesibilidad y también el transporte de madera, leña y productos agrícolas. Resultados similares han sido encontrados en otros estudios como son Abadía (2011), Henríquez-Dole (2012) y Díaz-Pacheco y Hewitt (2013), demostrando que las vías son causantes de las transformaciones de los paisajes. La Tabla 3 muestra los resultados de regresión logística que se obtuvieron entre las áreas transformadas y las variables biofísicas relieve, distancia a ríos y pendientes.
La Tabla 3 indica ajustes significativos en los modelos, donde el relieve y las pendientes al presentar un coeficiente positivo están mostrando que las transformaciones se acentúan en zonas altas y de bastante pendiente; esto se debe a que las condiciones de suelo son más apropiadas en estos lugares, lo que favorece para que se den procesos de transformación, ya sea para la agricultura y los pastos o también para el aprovechamiento de madera o leña.

En lo que respecta a la distancia a los ríos y quebradas, el modelo muestra buenos ajustes con coeficiente negativo, es decir, que las transformaciones se presentan en áreas cercanas a este componente biofísico. Este se explica en el sentido que el agua de las quebradas favorece para que se desarrollen actividades agropecuarias, como son los pastos y los cultivos. Resultados similares se han encontrado en otros ámbitos, como, por ejemplo, el estudio realizado por Gómez y Botero (2014), donde encontraron que las transformaciones del paisaje en las regiones del Bajo Cauca y Nordeste del departamento de Antioquia en Colombia fueron más acentuadas en zonas cercanas a los ríos.

TABLA 3. VALORES DE REGRESIÓN LOGÍSTICA ENTRE ÁREAS TRANSFORMADAS Y LAS VARIABLES: RELIEVE, DISTANCIA A RÍO Y PENDIENTES EN EL PÁRAMO DE PAJA BLANCA

\begin{tabular}{llccccc} 
& \multirow{2}{*}{ PÁRAMO } & \multicolumn{2}{c}{ RELIEVE } & \multicolumn{2}{c}{ DISTANCIA RÍOS } & \multicolumn{2}{c}{ PENDIENTES } \\
\cline { 2 - 8 } & Coeficientes & ROC & Coeficientes & ROC & Coeficientes & ROC \\
\hline Paja Blanca & 0,000527 & 0,78 & -0.00307 & 0,75 & 0,0390 & 0,82 \\
\hline
\end{tabular}

Fuente: Elaboración propia. 


\subsection{Simulación del paisaje del páramo de Paja Blanca para los próximos 30 años}

Una vez identificados y analizados los principales cambios de cobertura entre los periodos de 1984 y 2013, se procedió en primera instancia a la selección de variables, cuyo patrón espacial manifieste algún tipo de comportamiento que se crea que puede tener relación con el patrón de localización de cada uno de los cambios de coberturas que se identificaron. Las variables seleccionadas se indican en la tabla 4 con sus correspondientes valores del test de Cramer's V.

La Tabla 4 muestra que las pendientes, el relieve, la distancia a los ríos y las distancias a las viviendas aledañas ejercen cierto grado de influencia en la ubicación espacial de los mosaicos de pastos y cultivos. En las coberturas de páramo, las variables que ejercen mayor influencia fueron la pendiente y el relieve. El bosque natural denso no presentó relación con ninguna variable; y el bosque natural fragmentado manifestó tener influencia del relieve y la distancia a las viviendas.

\subsubsection{Transiciones potenciales}

Las transiciones más importantes de cambios de cobertura entre 1984 y 2013 fueron las siguientes:

- Bosque natural fragmentado (BNF) a mosaico de pastos y cultivos (MPC)

- Bosque natural fragmentado (BNF) a páramo (PA)

- Mosaico de pastos y cultivos (MPC) a bosque natural fragmentado (BNF)

- Páramo (PA) a bosque natural fragmentado (BNF).

Con estas transiciones, y considerando las variables anteriores, en el módulo LCM de Idrisi se realizaron los mapas potenciales de transición con interacciones que variaron entre 5.000 y 10000 , y niveles de precisión mayores al $80 \%$; los resultados se pueden apreciar en la Figura 7.

TABLA 4. ÍNDICE DE CRAMER'S V PARA LAS VARIABLES BIOFÍSICAS Y LAS DIFERENTES CATEGORÍAS DE COBERTURAS DEL SUELO EN EL PÁRAMO DE PAJA BLANCA

\begin{tabular}{lccccc}
\multirow{2}{*}{ COBERTURAS } & \multicolumn{5}{c}{ VARIABLES } \\
\cline { 2 - 6 } & PENDIENTE & RELIEVE & DISTRIOS & DISTVIAS & DISVIVIENDAS \\
\hline Mosaico de pastos y cultivos & 0,27 & 0,53 & 0,16 & 0,12 & 0,32 \\
Páramo & 0,17 & 0,5 & 0,08 & 0,08 & 0,13 \\
Bosque natural denso & 0 & 0,02 & 0 & 0 & 0 \\
Bosque natural fragmentado & 0,03 & 0,31 & 0,06 & 0,04 & 0,14 \\
\hline
\end{tabular}

Fuente: Elaboración propia. 

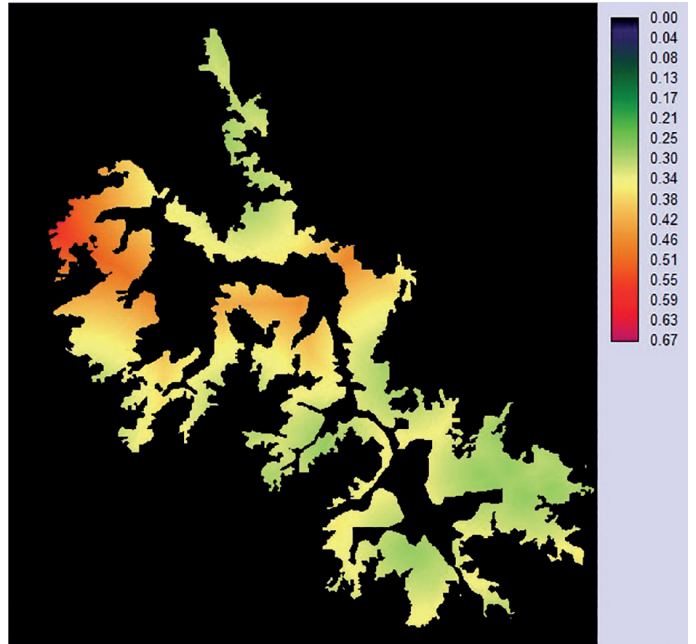

a)

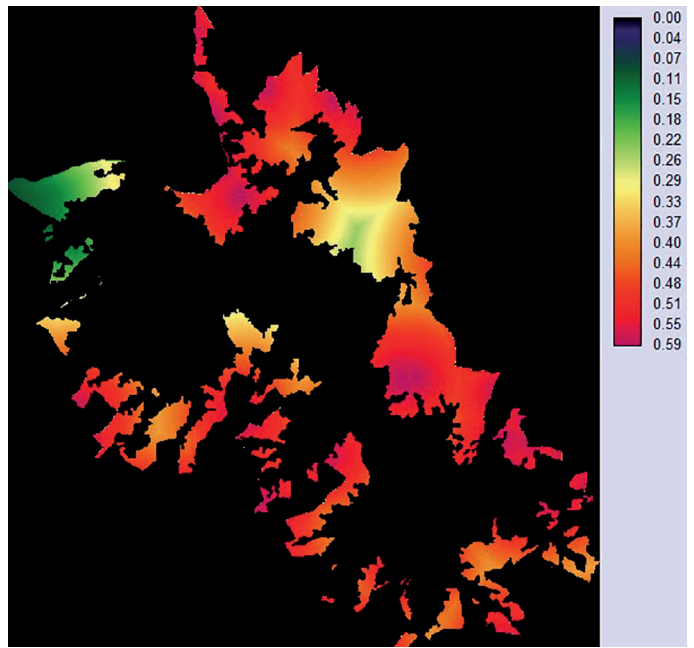

c)

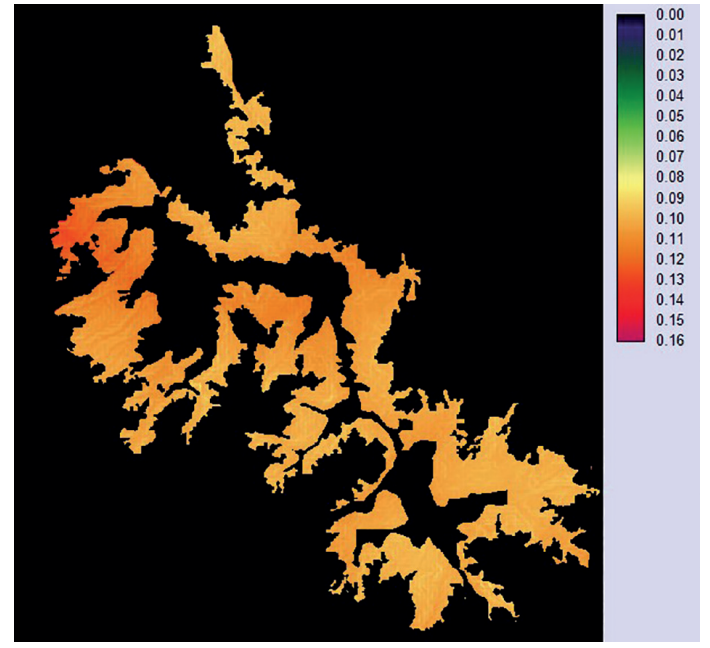

b)

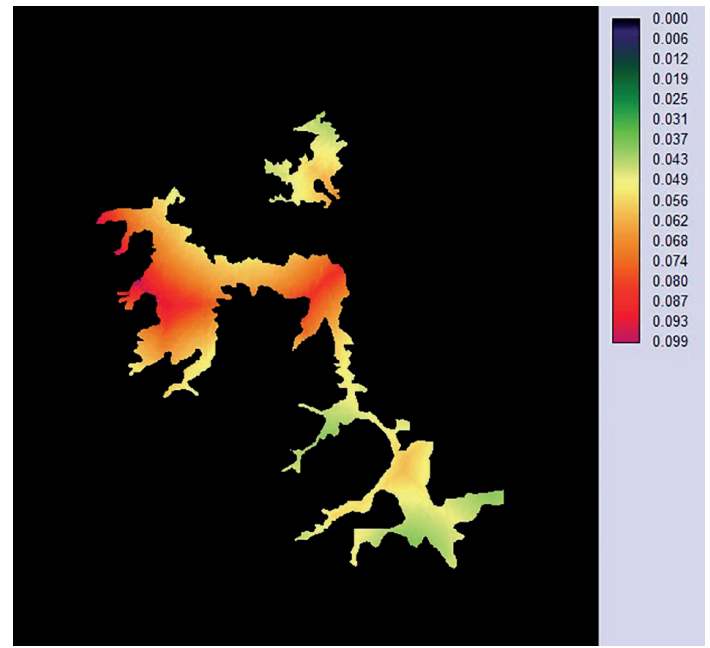

d)

\section{FIGURA 7.}

Mapas potenciales de transición de BNFMPC (a), BNF-PA (b), MPC-BNF (c) y PA-BNF (d), en el páramo de Paja Blanca, obtenidos en el módulo LCM de Idrisi

Fuente: Elaboración propia.
En estos mapas podemos observar que los valores del potencial en la escala de 0 a 1 son más amplios en la transición de BNF a MPC, y se acentúa hacia el sector occidental, en las veredas San José de Chillanquer y Villanueva, del municipio de Guachucal. Los valores de menos potencial se presentan para la transición PA-BNF.
Con los anteriores mapas potenciales de transición, en el programa Idrisi Selva, con el módulo LCM se simularon las coberturas para los próximos 30 años (1942); como resultado se ha obtenido una matriz de probabilidades de cambio (Tabla 5), una matriz de áreas de cambio (Tabla 6) y un mapa con la localización de las coberturas simuladas para el año 2042 (Figura 8). 
TABLA 5. MATRIZ DE PROBABILIDADES DE CAMBIO DE COBERTURAS DEL SUELO EN PÁRAMO DE PAJA BLANCA PARA EL PERIODO 2013-2042

\begin{tabular}{llccc}
\multicolumn{1}{c}{ CATEGORÍAS } & BND & BNF & MPC & PA \\
\hline Bosque natural denso (BND) & 0,7 & 0 & 0,3 & 0 \\
Bosque natural fragmentado (BNF) & 0,01 & 0,69 & 0,28 & 0,03 \\
Mosaico de pastos y cultivos (MPC) & 0 & 0,3 & 0,7 & 0 \\
Páramo (PA) & 0 & 0,22 & 0 & 0,78 \\
\hline
\end{tabular}

Fuente: Elaboración propia.

Observamos en la Tabla 5 que, para el año simulado, las probabilidades de permanencia de cada categoría de cobertura son casi más del $70 \%$; también se evidencia que existe una probabilidad del $28 \%$ y $30 \%$ de que las coberturas de BNF y BND respectivamente cambien a MPC, es decir, de continuar con las tendencias actuales, los bosques que aún predominan pueden llegar a alterarse por factores antrópicos. Sin embargo, se presenta una probabilidad del $30 \%$ de que algunas áreas de MPC cambien a BNF, lo cual demuestra que la tendencia a futuro es a que, mediante procesos de conservación, restauración ecológica y regeneración natural, se reconviertan áreas de uso antrópico a bosques naturales, lo cual es importante para la estabilidad ecológica del páramo.

La Tabla 6 indica las áreas (hectáreas) que posiblemente pueden cambiar de una categoría de cobertura a otra en el periodo simulado; en ella se evidencia que sumando las áreas de BND, BNF y PA que cambian a otras categorías mediante procesos de deforestación, obtenemos 486,8 ha afectadas antrópicamente, que corresponde a una tasa de deforestación anual de 16 has/año; es decir, la tendencia a futuro con el comportamiento actual es de mantenerse la misma tasa de deforestación anual que se presenta para el periodo 1984-2013. De esta manera, la simulación advierte que el páramo de Paja Blanca se mantendrá como un páramo clasificado según Cabrera y Ramírez (2014) como de baja transformación.

Se muestra también en la Tabla 6 casi 400 hectáreas de BNF transformadas a MPC, indicando que ahora que Paja Blanca ha sido declarado como Parque Natural Regional, sería de mucha importancia así como lo plantea Morales-Betancourt y Estévez (2006), que las instituciones encargadas el manejo del páramo adelanten programas y proyectos en los cuales sus habitantes no sean catalogados como "problema" o como "generadores de conflictos", sino que se los involucre en procesos de recuperación del ecosistema

TABLA 6. MATRIZ DE ÁREAS DE CAMBIO DE COBERTURAS DEL SUELO EN EL PÁRAMO DE PAJA BLANCA PARA EL PERIODO 2013-2042

\begin{tabular}{lcccc}
\multicolumn{1}{c}{ CATEGORÍAS } & BND & BNF & MPC & PA \\
\hline Bosque natural denso (BND) & 25,1 & 0 & 6 & 0 \\
Bosque natural fragmentado (BNF) & 14,1 & 1561,5 & 398,3 & 37,4 \\
Mosaico de pastos y cultivos (MPC) & 0 & 349,7 & 1353 & 0 \\
Páramo (PA) & 0 & 82,8 & 0 & 812,5 \\
\hline
\end{tabular}

Fuente: Elaboración propia. 
y en la educación ambiental de los visitantes. Estos autores manifiestan que "[...] debe reconocerse que los habitantes del páramo son parte integral de éste y deben ser entendidos como parte de la comunidad biótica paramuna, no como un accesorio que debe ser removido o del que puede prescindirse" (p. 44).

La Figura 8 presenta la ubicación espacial de las coberturas del suelo que se simularon para los próximos 30 años. Según este mapa, las áreas de BNF que se afectan por procesos antrópicos está más acentuada en los municipios de Guachucal y Sapuyes, especialmente en las veredas San José de Chllanquer, Villanueva y Maramba, localizadas al extremo noroccidental del páramo de Paja Blanca. También indica que en el municipio de Sapuyes, en las veredas La Campana y Los Monos, se presentan importantes áreas de MPC que se convierten a BNF; situación similar ocurre en la vereda Villa Sur del municipio de Ospina, y en la vereda Chorrera Negra del municipio del Cotandero.

Cabe resaltar que en el año 2015, dos años después de que se formuló el presente proyecto, el límite oficial de Paja Blanca, en su declaración oficial como Parque Natural Regional, fue diferente al tomado en este estudio, ya que el actual límite considera una superficie de 3.107 ha, es decir, se excluyeron todas las áreas de mosaico de pastos y cultivos, manteniéndose las áreas de bosque natural denso, bosque natural fragmentado y páramo. Esto quiere decir que los datos hallados en este estudio no dejan de evidenciar una situación preocupante al estimar 400 ha de bosque natural fragmentado que se encuentran en el actual límite que pasaran a ser mosaico de pastos y cultivos. De igual forma,

\section{FIGURA 8.}

Mapa de coberturas del suelo del páramo de Paja Blanca, simuladas para el año 2042.

Fuente: Elaboración propia.

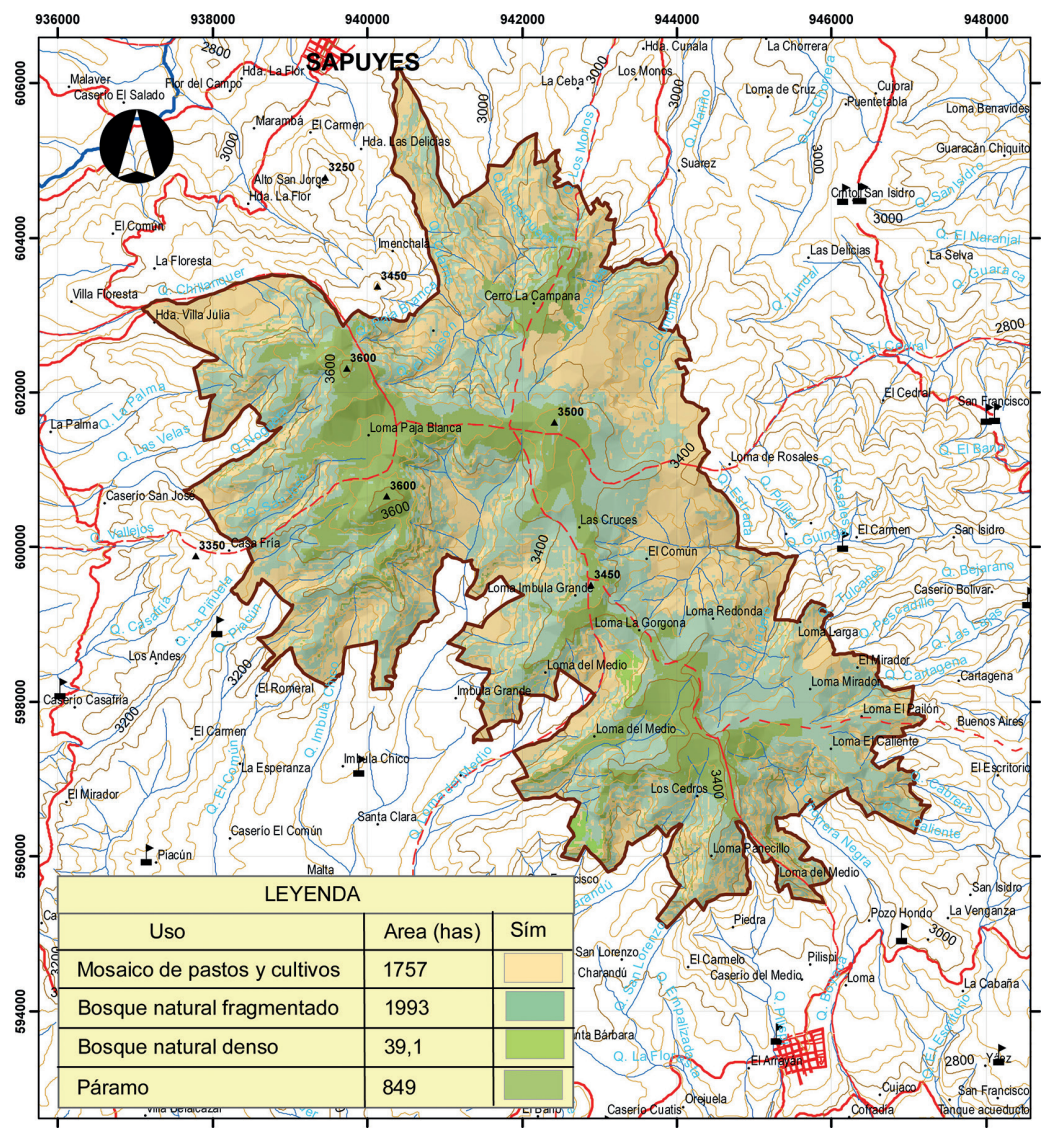


muestra la importancia de que los límites oficiales sean claramente delimitados en el terreno y, sobre todo, de que se adelante un programa de compra de predios para evitar su alteración limítrofe.

Desde el año 1997 hasta la fecha se han realizado tres (3) propuestas de delimitación, cada una de ellas condicionada por algunos factores fisicobióticos, especialmente las coberturas del suelo. Si no se establecen políticas claras y efectivas de conservación y manejo, es posible que dicho límite cada vez sea menor, como producto de los cambios en las coberturas que las simulaciones indican.

\subsubsection{Validación del modelo}

En vista de que no fue posible adquirir una imagen satelital actual (2016) para realizar un mapa y corroborar la exactitud del modelo, se decidió hacer un levantamiento de 50 puntos GPS en campo con el fin de registrar las coberturas observadas y con ello compararlas con un mapa simulado para el año 2016, a través de una matriz de confusión. La Tabla 7 indica los valores de la matriz de confusión, donde se puede apreciar una exactitud global del $76 \%$, y un índice de Kappa del 64\%, que según López de Ullibarri-Galparsoro y Pita-Fernández (1999) es considerado como bueno.

\section{Conclusiones}

En el periodo de 1984-2013, las transformaciones más sobresalientes corresponden al cambio de bosque natural fragmentado a mosaico de pastos y cultivos, y viceversa; indicando que hubo procesos de deforestación, pero también se presentó la reconversión de praderas y áreas agrícolas a bosques, que desde el punto de vista ambiental es bastante importante para la estabilidad del páramo.

La presencia de coberturas de páramo propiamente dicho, como son herbáceas y arbustivas, aproximadamente ocupa el $20 \%$ de todo el territorio de Paja Blanca, cuyas transformaciones o cambio hacia otras categorías no fueron significativas o alarmantes, pues solo se perdieron 78 hectáreas. Esto quizá sea un indicador de efectividad de las gestiones adelantadas por Corponariño en pro de la protección y conservación del páramo.

TABLA 7. MATRIZ DE CONFUSIÓN, PARA LAS COBERTURAS DEL PÁRAMO DE PAJA BLANCA SIMULADAS PARA EL AÑO 2016 Y DATOS RECOLECTADOS EN CAMPO

\begin{tabular}{lccccccc}
\hline \multicolumn{1}{c}{ COBERTURAS } & BND & BNF & MPC & PA & TOTAL & $\begin{array}{c}\text { EXAC } \\
\text { PRODUCT }\end{array} \begin{array}{c}\text { ERROR DE } \\
\text { OMISIÓN }\end{array}$ \\
\hline Bosque natural denso & 4 & 0 & 0 & 0 & 4 & 100,0 & 0,0 \\
Bosque natural fragmentado & 1 & 10 & 3 & 0 & 14 & 71,4 & 28,6 \\
Mosaico de pastos y cultivos & 0 & 2 & 19 & 4 & 25 & 76,0 & 24,0 \\
Páramo & 0 & 0 & 2 & 5 & 7 & 71,4 & 28,6 \\
Total & 5 & 12 & 24 & 9 & 50 & $(4+10+19+5) / 50 * 100=76$ \\
Exact usuario & 80,0 & 83,3 & 79,2 & 55,6 & & Exactitud global $=76 \%$ \\
Error comisión & & & & & & Índice de Kappa $=64 \%$ \\
\hline
\end{tabular}

Fuente: Elaboración propia. 
Con los resultados obtenidos en este estudio, se presenta una discrepancia con respecto a la tasa de deforestación anual que reporta Corponariño (2007b), de 11 has/año, ya que el análisis multitemporal realizado indica una pérdida de cobertura de bosque de 16 hectáreas al año, donde el factor antrópico es el causante de dicho comportamiento.

La densidad poblacional no mostró ser un factor causante de las transformaciones en el páramo de Paja Blanca, ni siquiera la distancia a las viviendas; pero se encontró una estrecha relación entre la distancia a las vías y áreas transformadas. Esto demuestra que las actividades antrópicas que causan el cambio no necesariamente provienen de quienes habitan directamente en el páramo. La tendencia futura del páramo de Paja Blanca es a mantenerse la misma tasa de deforestación (16 has/año), situación que lleva a pensar que se necesitan medidas de gestión más contundentes que logren bajar esta tasa.

El modelo de simulación obtenido presentó un buen ajuste y precisión, convirtiéndose en una herramienta muy importante para los procesos de toma de decisiones de quienes se encargan de la administración y gestión del páramo; sin embargo, se necesitan explorar otras variables socioeconómicas y políticas que puedan tener influencia en los cambios de cobertura, y que lleven a obtener un modelo con mucha mayor precisión. 


\section{Referencias}

Abadía, J.G. (2011). Cambios en la cobertura del paisaje y fuerzas conductoras en los Llanos Orientales Colombianos (Puerto López, Meta), 1988-2007 (tesis de pregrado). Pontificia Universidad Javeriana, Bogotá. Recuperado de https:// repository.javeriana.edu.co/handle/10554/12443

Aldana, J. (2011). Transformación físico-espacial de los paisajes boscosos del sector sur del Parque Nacional Natural los Katíos (1989-2010). Perspectivas Geográfica, 16. 31-54. Recuperado de revistas.uptc.edu.co/index.php/perspectiva/article/view/1749

Bertrand, G. (1968). Paysage et géographie physique globales: esquisse methodologique. Révue de Géographie des Pyrenées et Sud-Ouest, 39, 249. Recuperado de http:// www.persee.fr/doc/rgpso_0035-3221_1968_num_39_3_4553

Bonilla, A. (1985). Situación ambiental de Costa Rica. San José, Costa Rica: Ministerio de Cultura, Juventud y Deportes.

Cabrera, M. y Ramírez W. (Eds.). (2014). Restauración ecológica de los páramos de Colombia. Transformación y herramientas para su conservación. Bogotá: Instituto de Investigación de Recursos Biológicos Alexander von Humboldt (IAvH). Recuperado de http://humboldt.org.co/es/component/k2/item/562-restauracion-paramos

Chander, G. y Markham, B. (2003). Revised Landsat-5 TM radiometric calibration procedures and postcalibration dynamic ranges. IEEE Transactions on Geoscience and Remote Sensing, 41(11), 2674-2678. Recuperado de ieeexplore.ieee.org/ document/1245255

Chávez, P. S. (1996). Image-based atmospheric corrections-revisited and improved. Photogrammetric Engineering and Remote Sensing, 62, 1025-1036. Recuperado de https://www.researchgate.net/publication/236769129_Image-Based_ Atmospheric_Corrections_-_Revisited_and_Improved

Chuvieco, E. (1996). Fundamentos de Teledetección ( $3^{\mathrm{a}}$ ed.). Madrid: Ediciones RIALP.

Clark Labs. (2006). IDRISI Andes software. Worcester, M. A.: Clark University.

Cohen, J. (1960). A coefficient of agreement for nominal scales. Educational and Psychological Measurement, 20(1), 37-46. Recuperado de http://journals.sagepub. com/doi/abs/10.1177/001316446002000104

Congalton, R. Lunetta, R., Fenstermaker, L., Jensen, J.R., McWire, K. y Tinney, L. (1991). Remote sensing and geographic information system data integration: error sources and research issues. Photogram. Eng. Rem. Sensing, 57, 677-687.

Corporación Autónoma Regional de Nariño (Corponariño). (2007a). Plan de manejo páramo de Paja Blanca. Ipiales, Nariño: Corponariño.

Corporación Autónoma Regional de Nariño (Corponariño). (2007b). Tomo II. Informe final: Proyecto estado del arte de la información biofísica y Socioeconómica de los páramos de Nariño: caracterización biofísica de los páramos de Nariño. Nariño: Corponariño.

Corporación Autónoma Regional de Nariño (Corponariño). (2010). Declaratoria del parque natural regional páramo de Paja Blanca, territorio sagrado del pueblo de los Pastos. Nariño: Corponariño. 
Departamento Administrativo Nacional de Estadística (DANE). (2013).

Díaz-Pacheco, J. y Hewitt, R. (2013). Modelado del cambio de usos de suelo urbano a través de redes neuronales artificiales. Comparación con dos aplicaciones de software. GeoFocus, 14, 1-22. Recuperado de geofocus.rediris.es/2014/ Articulo1_2014.pdf

Gómez-Zotano, J. y Riesco, P. (2010). Marco conceptual y metodológico para los paisajes españoles. Aplicación a tres escalas espaciales. Sevilla: Consejería de Obras Públicas y Vivienda.

Gómez, L. y Botero, V. (2014). Análisis comparativo de dos modelos de deforestación: redes neuronales artificiales y regresión logística. XVI Simposio Internacional SELPER. Medellín, Colombia. 23 p.

Hartshorm, G. (1983). Costa Rica perfil ambiental. San José, Costa Rica: Trejos.

Henríquez-Dole. L. (2012). Escenarios futuros de uso de suelo para el análisis del efecto del cambio global en los recursos hídricos aplicado al acuífero de la Mancha Oriental (tesis de maestría). Universitat Politècnica De València, España.

Isasi, N. y Galván, M. (2004). Redes Neuronales Artificiales: un enfoque práctico. Madrid: Pearson Education, S. A.

López de Ullibarri-Galparsoro I. y Pita-Fernández, S. (1999). Medición de concordancia: Índice de Kappa. Cad Aten Primaria, (6), 169-171. Recuperado de http://www.fisterra.com/mbe/investiga/kappa/kappa2.pdf

Morales-Betancourt, J. y Estévez-Varón, J. (2006). El páramo: ¿ecosistema en vía de extinción? Revista Lunazul, 22, 1-13. Recuperado de https://isfcolombia.uniandes. edu.co/images/documentos/paramoextinsion.pdf

Pérez, S. y Protti, F. (1978). Comportamiento del sector forestal durante el período 19501977. San José: Oficina de Planificación Sectorial Agropecuaria.

Pineda-Jaimes, N., Bosque-Sendra, J., Gómez-Delgado, M. y Plata-Rocha, W. (2009). Análisis de cambio del uso del suelo en el Estado de México mediante sistemas de información geográfica y técnicas de regresión multivariantes. Una aproximación a los procesos de deforestación. Investigaciones Geográficas. Boletín del Instituto de Geografía, 69, 33-52. Recuperado de http://www.scielo.org.mx/ pdf/igeo/n69/n69a4.pdf

Sandoval, V. y Oyarzun, V. (2004). Modelamiento y prognosis espacial del cambio en el uso del suelo. Quebr@cho: Revista de Ciencias Forestales, 11, 9-21. Recuperado de http://fcf.unse.edu.ar/archivos/quebracho/01-sandoval-oyarzun-q11.pdf

Thome, K., Palluconi, F., Takashima, T. y Masuda, K. (1998). Atmospheric correction of ASTER. IEEE Transactions on Geoscience and Remote Sensing, 36(4), 1199-1211.

Wooster, M.J. (1996). Orbit calibration of the ATSR1 $1.6 \mathrm{~mm}$ channel using high resolution data from the JERS1 (Fuyo1) optical sensor. International Journal of Remote Sensing, 17(5), 1069-1074.

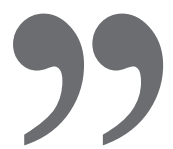

